\title{
Second neoplasm: 31-year latency after childhood leukemia
}

\author{
Amit Nautiyal MD, Pooja Chaukiyal MD
}

Previously published at www.cmaj.ca

A 33-year-old man presented with a six-week history of headache, nausea, gait imbalance and changes in personality that included apathy and disinhibition. The patient had a history of childhood acute lymphoblastic leukemia that had been diagnosed when he was two years old. He had been given vincristine, prednisone and L-asparaginase at that time, and had received 10 fractions of $1800 \mathrm{cGy}$ craniospinal irradiation. The disease had gone into clinical remission and the patient had been lost to oncologic follow-up.

A magnetic resonance imaging scan of our patient's brain showed a right frontal mass, marked vasogenic edema and a prominent midline shift (Figure 1). A right frontal craniotomy was performed with intraoperative frozen section and complete resection of the tumour. The final histopathologic diagnosis, to our surprise and relief, was a meningioma with mixed meningothelial and angiomatous features.

Survivors of childhood acute lymphoblastic leukemia, particularly those who have received cranial irradiation, are at substantial risk for a second malignancy. ${ }^{1}$ Brain tumours, most commonly meningiomas, are among the most common second neoplasms discovered in these patients. ${ }^{1}$ Other tumours of the central nervous system, including astrocytomas, gliomas, medulloblastomas and nonspecified tumours, have been reported.

Over 14000 patients diagnosed with childhood and adolescent cancer between 1970 and 1986 have been followed by the Childhood Cancer Survivor Study established in 1994. The 20 -year cumulative incidence of second neoplasms was reported in 2001 to be $3.2 \%{ }^{3}$ By 2008, the occurrence of second malignant neoplasms had increased 2.3-fold, from 314 second malignant neoplasms in 298 patients to 802 in 730 patients. $^{4}$ The 30-year cumulative incidence of second neoplasms was $9.3 \%$ - an increase of $6.1 \%$ over the 20 -year incidence.

As shown in our patient, survivors of childhood leukemia can develop second neoplasms many years after their initial cancer has been effectively treated. Such patients therefore require appropriate counselling and long-term follow-up. ${ }^{3}$

From the Department of Medicine (Nautiyal), University of Pittsburgh; and the Department of Medicine (Chaukiyal), Veterans Affairs Pittsburgh Health Care System, Pittsburgh, USA

CMAJ 2009. DOI:10.1503/cmaj.090562

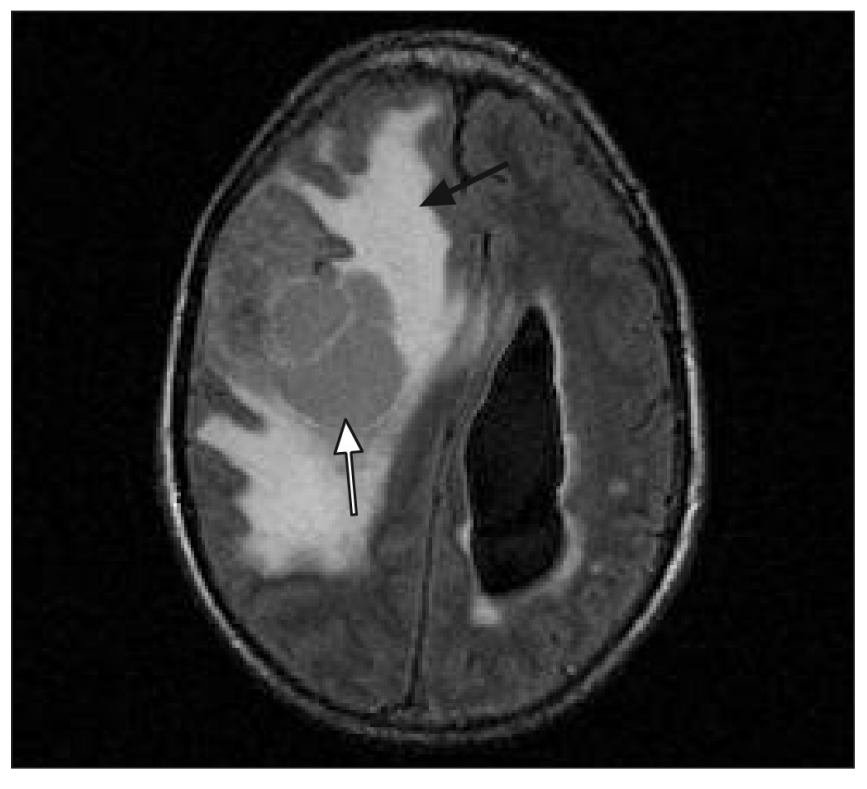

Figure 1: Magnetic resonance imaging scan of the brain of a 33-year-old survivor of childhood leukemia, showing a right frontal mass (white arrow). Marked vasogenic edema (black arrow) and a prominent midline shift are visible.

Because the second neoplasm may be distinct from the original tumour, prognostication should always await final diagnosis based on tissue analysis. Our patient recovered uneventfully and was discharged on the fourth postoperative day.

This article has been peer reviewed.

Competing interests: None declared.

\section{REFERENCES}

1. Maniar TN, Braunstein I, Keefe S, et al. Childhood ALL and second neoplasms. Cancer Biol Ther 2007;6:1525-31.

2. Mody R, Li S, Dover DC, et al. Twenty-five-year follow-up among survivors of childhood acute lymphoblastic leukemia: a report from the Childhood Cancer Survivor Study. Blood 2008;111:5515-23.

3. Neglia JP, Friedman DL, Yasui Y, et al. Second malignant neoplasms in five yea survivors of childhood cancer: report from the Childhood Cancer Survivor Study. J Natl Cancer Inst 2001;93:618-29.

4. Meadows AT, Friedman DL, Neglia JP, et al. Second neoplasms in survivors of childhood cancer survivor study cohort. J Clin Oncol 2009;27:2356-62. 